\title{
ESTUDIO FITOQUÍMICO PRELIMINAR Y EVALUACIÓN DE LA ACTIVIDAD CITOTÓXICA DEL LÁTEX DE EUPHORBIA LAURIFOLIA JUSS. EX. LAM SOBRE ARTEMIA SALINA
}

\section{Preliminary Phytochemical Study and Evaluation of Cytotoxic Activity of Euphorbia laurifolia Juss. ex. Lam latex on Artemia salina}

\author{
Aida Miranda*, Diego Vinueza, Karen Acosta, Cecilia Toaquiza, \\ Estela Mendez, Fabian Arias
}

Facultad de Ciencias, Escuela Superior Politécnica de Chimborazo, Panamericana Sur km 1 1/2, Riobamba-Ecuador. aidita_6799@hotmail.es

\section{$\mathbf{R}$}

Euphorbia laurifolia es una especie vegetal de la familia Euphorbiaceae distribuida en Colombia, Venezuela y Ecuador. Tradicionalmente es usada para el tratamiento de afecciones al hígado, para evitar la formación de abscesos y verrugas en la piel. Debido a su alta disponibilidad y variedad de usos constituye una materia prima de importancia fitoquímica y farmacológica. El objetivo de esta investigación fue realizar el estudio fitoquímico preliminar y la evaluación de la actividad citotóxica del látex de E. laurifolia sobre Artemia salina. Se obtuvieron diversos extractos del látex de la especie, a partir de los cuales se realizó la separación e identificación mediante Cromatografía en Capa Fina (TLC). La evaluación de la actividad citotóxica sobre Artemia salina se llevó a cabo utilizando la fracción hexánica usando concentraciones de 0,31, 62, 125, 250, 500, 1000 ppm. Se estableció que E. laurifolia presenta compuestos terpénicos, identificando por TLC posibles moléculas como el borneol (Rf 0.24) y el cineol (Rf 0.40). Respecto a la citotoxicidad de la fracción hexánica, se obtuvo una concentración letal media (LC50) de 214.50 ppm, demostrando que el extracto es tóxico. El látex de E. laurifolia es rico en compuestos terpénicos con alta actividad citotóxica; por tanto, presenta un destacable potencial para futuros estudios farmacológicos.

Palabras claves: Euphorbia laurifolia, actividad citotóxica, Artemia salina, látex, terpenos, Euphorbiaceae

\section{A}

Euphorbia laurifolia is a plant species that belongs to the family Euphorbiaceae located in Colombia, Venezuela and Ecuador. Traditionally, it is used for liver affections and to avoid the formation of skin abscesses and warts. Due to its high availability and variety of uses, it has phytochemical and pharmacological importance. The aim of this investigation was to carry out preliminary phytochemical study and evaluation of the cytotoxic activity of E. laurifolia latex on Artemia salina. Several latex extracts, separation and identification of compounds was performed by Thin Layer Chromatography (TLC). The evaluation of the cytotoxic activity on Artemia salina was carried out using the hexanic fraction at concentrations of $0,31,62,125,250,500,1000 \mathrm{ppm}$. It was established that E. laurifolia contains terpene compounds, identified by TLC molecules such as borneol ( $\operatorname{Rf} 0.24)$ and cineole (Rf 0.40). Regarding the cytotoxicity of the hexane fraction, the lethal concentration (LC50) was 214.50 $\mathrm{ppm}$, showing that the extract is toxic. The latex of E. laurifolia is rich in terpene compounds with 
high cytotoxic activity; therefore, it presents a remarkable potential for future pharmacological studies.

Keywords: Euphorbia laurifolia, cytotoxic activity, Artemia salina, latex, terpenes, Euphorbiaceae.

\section{INTRODUCCIÓN}

La familia Euphorbiaceae corresponde al vegetal más extenso de las angiospermas, muy utilizada en la medicina tradicional e incluye casi 8000 especies (1). Los diterpenoides del género Euphorbia presentan actividades irritantes en la piel y ojos (2) (3), actividad apoptótica (4) y citotóxica en células (5).

La familia Euphorbiacea se caracteriza por sus hojas glabras, estípulas ausentes y numerosas flores masculinas rodeando una flor femenina central pedicelada y de forma particular segrega un látex lechoso (6). El látex es utilizado para el tratamiento de afecciones del hígado en forma de emplastos, formación de abscesos y verrugas en la piel. (7).

Se ha evaluado la efectiva capacidad molusquicida de varias especies de Euphorbiaceae, entre ellas la E. pulcherrima y E. hirta contra caracoles Lymnaea acuminata (8), E. splendens y E. aphylla contra Biomphalaria alexandrina (9) y la E. helioscopia con la E. schimperiana contra Biomphalaria pfeifferi

La especie Euphorbia laurifolia es un arbusto presente en la zona andina de Venezuela, Colombia y Ecuador a 1000 y 3000 msnm. En el género Euphorbia se ha identificado diferentes compuestos terpénicos como antiquol C, antiquol B, euphorbol, camelliol C (11). Ávila, L, et al, (2010) identificaron compuestos triterpénicos como el lanosterol y la latazienona, además de un nuevo compuesto diterpénico macrocíclico tipo latirano. La actividad viral de la E. laurifolia fue estudiada frente al virus de la inmunodeficiencia humana tipo $1 \mathrm{VIH}$ 1 (1) y su actividad molusquicida contra Biomphalaria glabrata (12) presentando resultados favorables en ambos estudios.

Al considerar los estudios previos el objetivo del presente trabajo fue determinar la actividad citotóxica de la especie Euphorbia laurifolia frente Artemia salina, con el fin de proporcionar una base científica para futuros análisis antimicrobianos.

\section{MATERIALES Y MÉTODOS}

\section{Material vegetal}

Se utilizó el látex de Euphorbia laurifolia, el cual fue recolectado en la parroquia Cubijíes, provincia de Chimborazo, (latitud: -1.65 longitud: -78.5833). La zona de recolección presenta un clima generalmente frio, con temperatura que oscila entre de $12{ }^{\circ} \mathrm{C}$ y $16^{\circ} \mathrm{C}$ y una altitud de $2503 \mathrm{~m} \mathrm{~s}$. n. m. La especie fue identificada por el Ingeniero Jorge $\mathrm{Ca}$ ranqui, responsable del herbario de la Facultad de Ciencias-ESPOCH (Est. Espoch: Carolina Huaraca 2899)

\section{Material biológico: Artemia salina}

El material biológico fue adquirido en estadío de huevos, los cuales fueron incubados en condiciones de agua salada a una temperatura entre $25-32{ }^{\circ} \mathrm{C}$ hasta su eclosión.

\section{Obtención de extractos}

El látex segregado de E. laurifolia fue obtenido a través de un corte transversal en las axilas iniciales de las ramas, en dichos cortes se presenta una emanación de secreción la cual fue recolectada en metanol. La solución alcohólica se dejó en maceración por 24 horas en un lugar fresco y seco, en ausencia de luz. El látex macerado se filtró a través de una membrana de $11 \mu \mathrm{m}$ de porosidad. El filtrado metanólico fue concentrado a $40{ }^{\circ} \mathrm{C}$ hasta sequedad; se reconstituyó con hexano con la finalidad de separar compuestos apolares de los polares. La fase hexánica se concentró (Fr 1) y la 


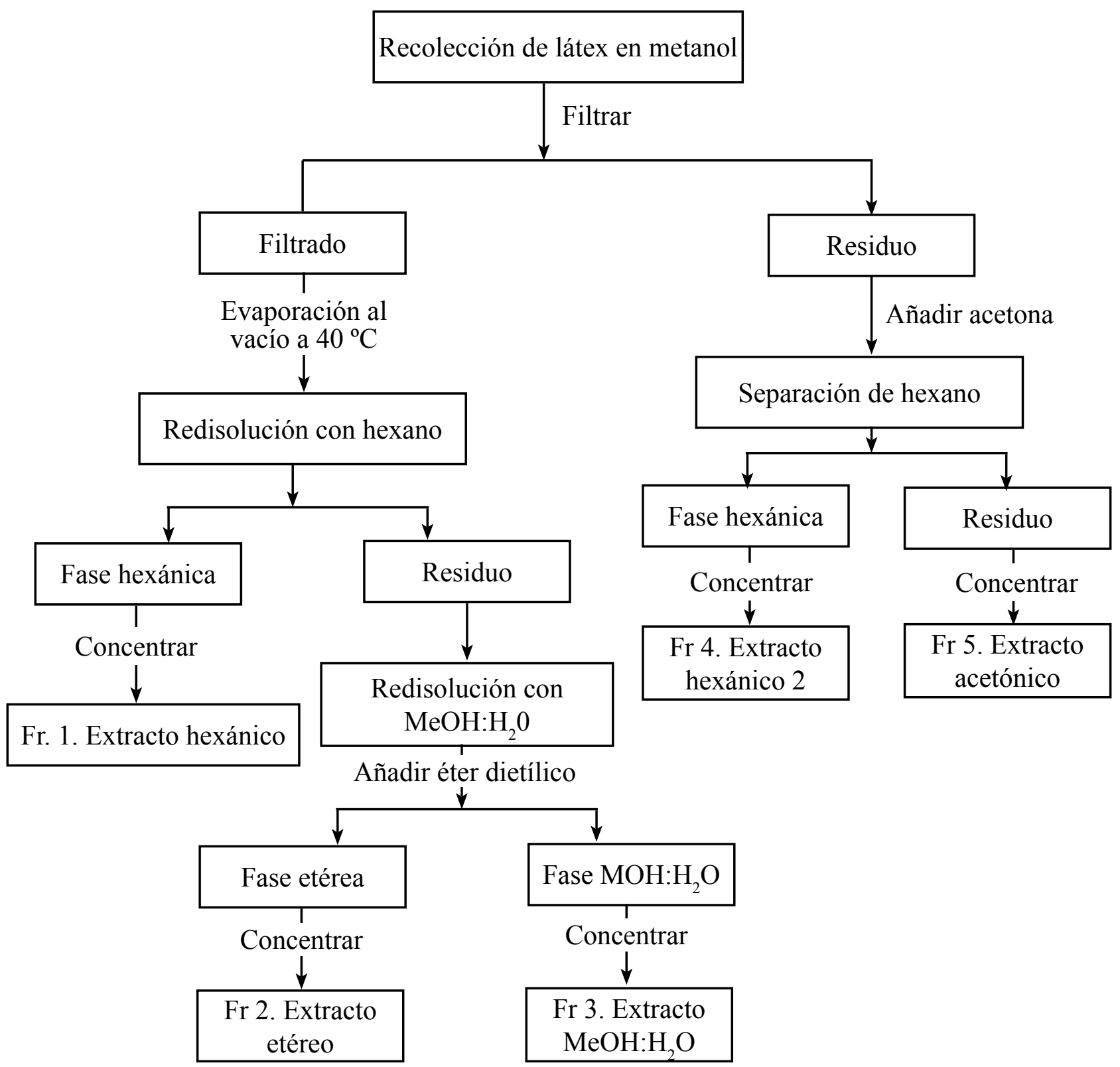

Figura 1. Proceso de obtención de los extractos del látex Euphorbia laurifolia

parte insoluble en hexano se reconstituyó con $\mathrm{MeOH}: \mathrm{H}_{2} \mathrm{O}(1: 1)$, a la cual se añadió éter para la separación de posibles compuestos apolares restantes. Tanto la fase $\mathrm{MeOH}: \mathrm{H}_{2} \mathrm{O}(\mathrm{Fr} 3)$ como la etérea (Fr 2) fueron concentradas (13). La fase sólida filtrada fue disuelta en acetona, añadiendo un volumen proporcional de hexano para la separación de compuestos apolares. La fase hexánica (Fr 4) y acetónica (Fr 5) fueron separadas y concentradas hasta sequedad. El proceso de obtención de los extractos se detalla en la figura 1.
El objetivo de realizar cinco extracciones fue identicar extractos que contengan mayor cantidad de compuestos terpénicos

\section{Separación e identificación de compuestos por cromatografía en capa fina (TLC)}

Cada uno de los extractos obtenidos anteriormente fueron sometidos a cromatografía en capa fina (TLC) empleando placas de sílice gel 60F 254 de $10 \mathrm{~cm} \mathrm{x} 4 \mathrm{~cm}$. Se usó como fase móvil tolueno y acetato de etilo en una relación (7:3) (2). Se observó bajo la luz UV los componentes terpénicos y posteriormente se reveló con ácido sulfúrico-vainillina (50:50) sometiéndolas al calor por dos minutos. 


\section{Determinación de LC50 de la fracción hexánica}

\section{Eclosión de A. salina}

Los huevos de $A$. salina se incubaron durante $48 \mathrm{~h}$ en una solución $\mathrm{NaCl} 1 \%$ exenta de nitrato, fosfato y silicato a una temperatura controlada entre $26-30{ }^{\circ} \mathrm{C}$, en presencia de una lámpara de tungsteno. Durante el tiempo de incubación, la solución fue oxigenada a través de una bomba de aire.

\section{Ensayo de letalidad}

Para el bioensayo solamente se utilizó el extracto hexánico (Fr 1), debido a que el extracto etéreo dio problemas en la solubilidad.

Para la preparación de la solución madre del extracto hexánico se pesaron $0,025 \mathrm{~g}$ de extracto, se disolvieron en $0,25 \mathrm{ml}$ de dimetil sulfoxido (DMSO) y se aforaron a $25 \mathrm{~mL}$ con solución de $\mathrm{NaCl} 0,1 \%$. Para la obtención del blanco, se disolvieron $0,25 \mathrm{~mL}$ de DMSO en $25 \mathrm{~mL}$ de $\mathrm{NaCl} \mathrm{0,1 \% .}$

La fracción hexánica fue preparada a concentraciones de $0 ; 31 ; 62 ; 125 ; 250 ; 500 ; 1000$ ppm del extracto en $\mathrm{NaCl} 0,1 \%$. Cada una de las diluciones fueron colocadas en tubos de ensayo donde fueron liberadas 10 larvas, sumergiéndolas en la solución para evitar el contacto con el aire (18). Después de 24 h, se determinó el porcentaje de letalidad a cada concentración y se interpretó: 0-10\% no tóxico, 11-50 \% moderadamente tóxico, 51-90 $\%$ altamente tóxico y $100 \%$ extremadamente tóxico (22). Todos los ensayos se realizaron por triplicado.

Además, los datos obtenidos se tabularon a través del software estadístico Análisis Probit TSK para la determinación del nivel LC50 (17).

El extracto vegetal se considera tóxico si presenta un valor de LC50 menor de 1000 ppm mientras que no es activo si es mayor a $1000 \mathrm{ppm}$ (23).

\section{RESULTADOS}

Separación e identificación de compuestos por cromatografía en capa fina (TLC)

Al observar las placas de TLC, de las cinco fracciones solamente se evidenciaron compuestos en la Fr1 y Fr2. En las placas de TLC del extracto hexánico (figura 2) se evidencian siete manchas que indican la posible presencia de compuestos y en el TLC del extracto etéreo trece

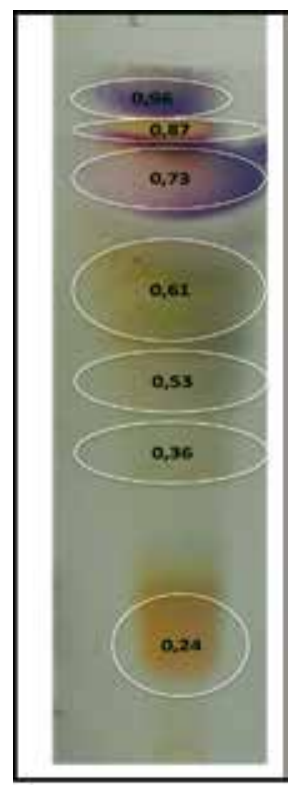

Figura 2. Cromatografía en Capa Fina del extracto hexánico

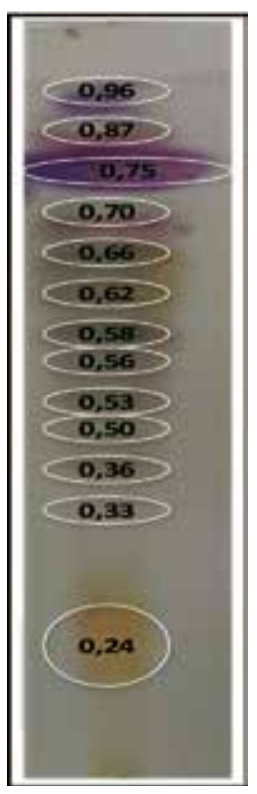

Figura 3. Cromatografía en Capa Fina del extracto etéreo

(figura 3), las cuales presentan coloraciones y factores de retención (Rf acrónimo en inglés) similares.

Las coloraciones observadas en el revelado se deben al revelador utilizado (ácido sulfúrico-vainillina), el cual al reaccionar con un grupo fenol forma manchas de colores naranja, amarillo, 
marrón y morado. Según Ivor Smith (1960), cada color tomado por los compuestos en las placas de TLC se debe a que la reacción inicial sería la sustitución electrofílica aromática de un fenol. Los terpenos reaccionan con el ácido sulfúrico formando compuestos oxidados y que en presencia de vainillina forma cromógenos. Los cromógenos revelados en ambas placas presentan similitud cromática por lo que se determina que en ambas extracciones se lograron extraer compuestos similares; sin embargo, en la placa del extracto etéreo se evidencia la presencia de cinco compuestos adicionales en comparación con la placa del extracto hexánico. Esto se debe a que el hexano extrae principalmente compuestos hidrófobos poco o nada polares con una lipofilia extremadamente alta, de los cuales se esperan compuestos neutros poco polares, esteroides y ácidos grasos de alto contenido en carbono (15). En cambio, el éter dietílico extrae principalmente compuestos hidrofóbicos medios (incluidos compuestos ácidos y básicos neutros de mediana y baja polaridad), de los cuales se esperan esteroides, ceras, ácidos grasos, alcaloides y polímeros carbonatados de cadena polar (15).

Los valores de $\mathrm{Rf}$ determinados en ambas placas se consideran como "Rf. Al comparar las distancias de recorrido de cada placa TLC se determinó que existen coincidencias en ambas placas en los compuestos que se encuentran en el rango de $\operatorname{Rf} 0,24,0,36,0,53,0,87,0,96$., identificando posibles compuestos terpenoides como el borneol (Rf 0,24) y el cineol (Rf 0,40) (16).

En el resto de fracciones (Fr3, Fr 4, Fr5) no se identificaron compuestos terpénicos al someterlas a las mismas condiciones.

\section{Determinación de LC50 de la fracción hexánica}

Los resultados del efecto citotóxico del extracto hexánico del látex de Euphorbia laurifolia frente a A. Salina se encuentran detallados en la Figura 4 y Tabla 1, donde se observa que al aumentar la concentración del extracto existe igualmente un aumento de la mortalidad del crustáceo, llegando hasta una disminución poblacional máxima del $78 \%$ a una concentración de $1000 \mathrm{mg} / \mathrm{l}$. A concentraciones inferiores a $200 \mathrm{ppm}$ se puede observar que el extracto es moderadamente tóxico, mientras que a concentraciones superiores a $200 \mathrm{ppm}$ el extracto es altamente tóxico (22).

Por otro lado, el control, el cual presenta DMSO y no contiene extracto, presenta porcentajes de mortalidad inferiores al $10 \%$, interpretándose como no tóxico (22). El hecho de que se provoque cierta mortalidad sobre los nauplios puede deberse a su gran propiedad transdérmica, lo que podría provocar hipertonicidad en la A. salina (19).

La determinación de la dosis letal(LC50) a través del software estadístico Análisis Probit TSK se realizó con los valores promedios de los ensayos (figura 5), obteniendo un valor de $214.50 \mathrm{ppm}$ a un nivel de confianza del $95 \%$, lo cual indica que el extracto hexánico del látex de E. laurifolia es tóxico. Este resultado muestra el potencial de esta especie vegetal como citotóxica y como un posible agente antitumoral, siendo necesarios más estudios al respecto (23).

La citotoxicidad de la fracción hexánica de E. laurifolia fue menor en comparación con Neoboutonia macrocalyx

\begin{tabular}{|c|c|c|c|c|}
\hline $\begin{array}{c}\text { Concentración } \\
\mathbf{m g} / \mathbf{L}\end{array}$ & $\begin{array}{c}\text { Total larvas } \\
\text { vivas }\end{array}$ & $\begin{array}{c}\text { Mortalidad (\%) } \\
\text { Ensayo 1 }\end{array}$ & $\begin{array}{c}\text { Mortalidad (\%) } \\
\text { Ensayo 2 }\end{array}$ & $\begin{array}{c}\text { Mortalidad (\%) } \\
\text { Ensayo 3 }\end{array}$ \\
\hline 0 & 100 & 7 & 6 & 9 \\
\hline 31 & 100 & 13 & 15 & 12 \\
\hline 62 & 100 & 33 & 30 & 35 \\
\hline 125 & 100 & 50 & 52 & 55 \\
\hline 500 & 100 & 63 & 66 & 65 \\
\hline 1000 & 100 & 73 & 78 & 72 \\
\hline
\end{tabular}

Mortalidad: \% de larvas muertas

Concentración: $\mathrm{mg} / \mathrm{L}$ de extracto 


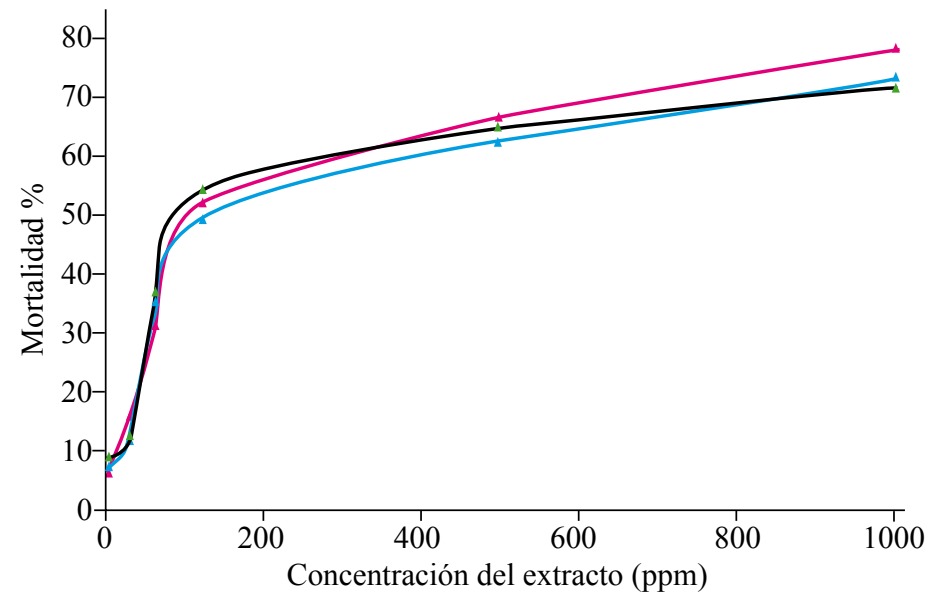

Figura 4. Porcentaje de mortalidad del extracto hexánico de Euphorbia laurifolia sobre Artemia salina

$(\mathrm{LC} 50=0.6 \mathrm{ppm})$, especie también perteneciente a la familia Euphorbiaceae (23). Igualmente, E. laurifolia fue menos citotóxica que Euphorbia splendens (LC50=40 ppm) y Euphorbia aphylla (LC50=87,6 ppm) contra Biomphalaria alexandrina (9). Por otra parte, Euphorbia helioscopia y Euphorbia schimperiana presentaron valores de LC50 entre 34-66 ppm frente a Biomphalaria pfeifferi (10).
Los terpenos son compuestos naturales que muestran un amplio espectro de efectos biológicos como la citotoxicidad. Son capaces de inhibir la vida de células neoplásicas y producir apoptosis en células cancerosas, sin destruir las células normales del organismo (20). Asimismo, estos metabolitos son considerados como fitoalexinas que aumentan su concentración en las especies vegetales al verse afectadas por algún agente externo (virus, bacterias, hongos) (21).

\section{CONCLUSIONES}

El látex de E. laurifolia presentó una destacable diversidad de compuestos terpénicos, identificando posibles compuestos como el borneol y el cineol. La fracción hexánica del látex de la especie en estudio presentó una notable toxicidad frente a Artemia salina, lo cual es un indicio preliminar de su citoxicidad y su potencial como agente antiparasitario

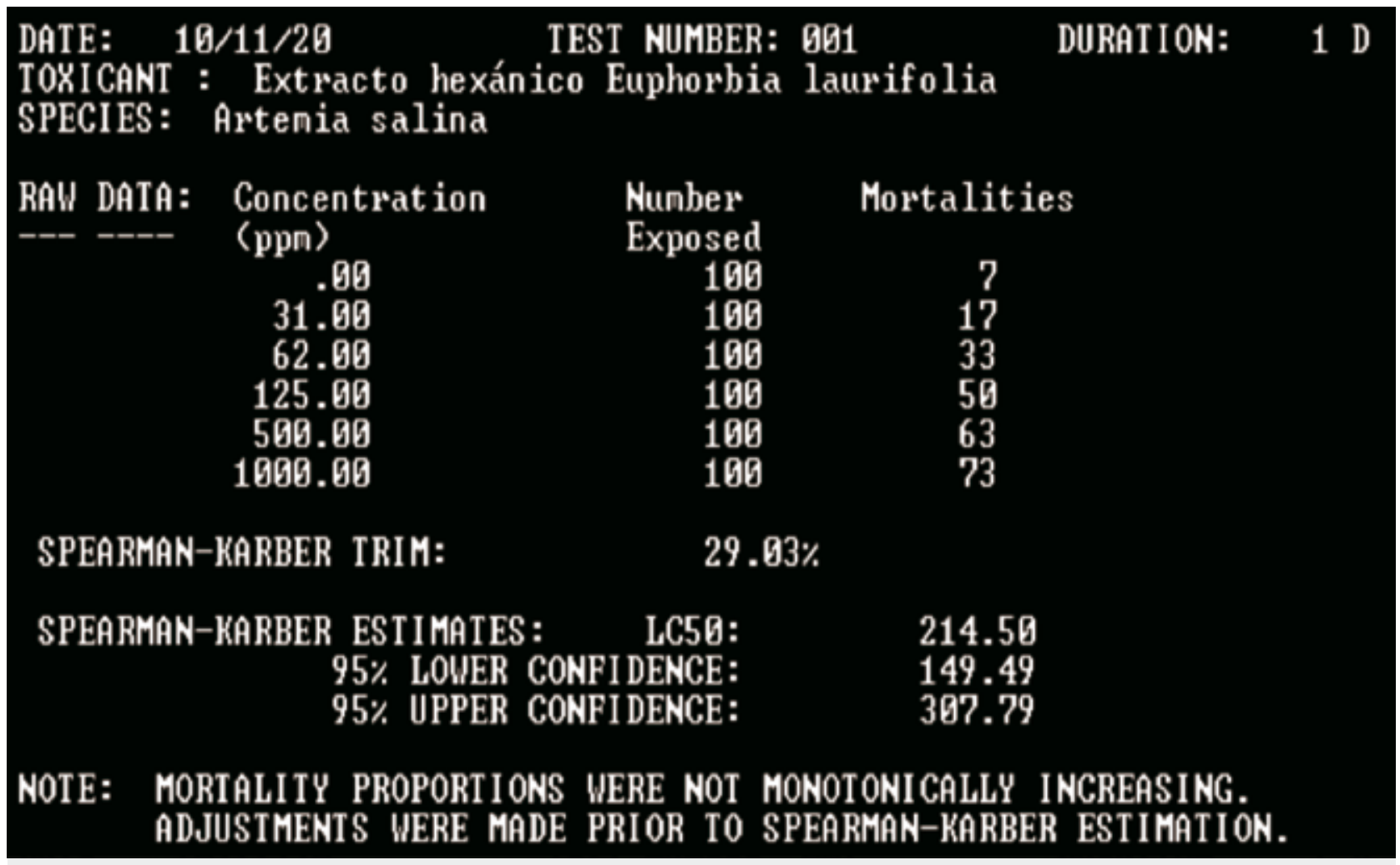

Figura 5. Resultados del LC50 a través del software estadístico Análisis Probit TSK 
y/o antitumoral de importancia farmacológica. Es necesaria la realización de otros estudios de toxicidad y de actividad biológica, con el fin de establecer su seguridad y eficacia en el tratamiento de enfermedades.

\section{AGRADECIMIENTOS}

A los autores que han contribuido en la investigación y redacción del texto.

1. Ávila L, Pérez M, Sánchez-Duffhues G, Hernández-Galán R, Muñoz E. Effects of Diterpenes From Latex of Euphorbia lactea and Euphorbia laurifolia on Human Immunodeficiency Virus Type 1 Reactivation. Phytochemistry. 2010; 7: 243-248

2. Ahmed, A, Couladis, M., Mahmoud, A., De Adams, A., Mabry, T. Ingol diterpene ester from the latex of Euphorbia lactea. Fitoterapia.1999; 70: 140-143.

3. Lu Z, Yang M, Zhang J, Chen G, Huang, H., Guan S, Ingenane diterpenoids from Euphorbia esula. Phytochemistry. 2008; 69: 812-819.

4. Blanco-Molina M., Tron G, Macho A, Lucena C, Calzado M, Muñoz E, Appendino, G., Ingenol Esters Induce Apoptosis in Jurkat Cells Through an AP 1 and NF-UB Independent Pathway. 2001; 8: 767-778

5. Vigone A, Tron G, Surico D, Baj G, Appendino G, Surico N. Ingenol Derivatives Inhibit Proliferation and Induce Apoptosis in Breast Cancer Cell Lines. Eur. J. Gynaecol. Oncol. 2005; 26, 526-530.

6. Jijón C. Euphorbia laurifolia. [Internet]. 2014 [citado 12 noviembre 2014]; http://plantasnativas. visitavirtualjbq.com/index.php?option $=$ com content\&view $=$ article $\& i d=13$ :euphorbia-laurifolia\&cati$\mathrm{d}=14$ :siglo-xviii-coleccion-de-joseph-de-jussieu\&Itemid $=108$

7. Ulloa C, Moller P. Árboles y arbustos de los Andes. [Internet].2014[citado 11 noviembre 2014],http:// www.efloras.org/florataxon.aspx?flora id=201\&taxon id=112355

8. Singh SK, Yadav RP, Singh, D, Singh, A. Toxic Effect of Two Common Euphorbiales latices on The Freshwater Snail Lymnaea acuminate. Enviro Toxicol Pharmacol. 2004; 15: 87-93.

9. Hassan AA, Mahmoud AE, Hassan RA, Huseein EAM. Evaluation of Euphorbia aphylla, Ziziphus spina-christi and Enterolobium contortisiliquum as Molluscicidal Agents. J Am Sci. 2011; 7: 511-20.

10. Al-Zanbagi NA, Banaja AEA, Barrett, J. Molluscicidal Activity of Some Saudi Arabian Euphorbiales Against the Snail Biomphalaria pfeifferi. J Ethnopharmacol. 2010; 70: 119-25.

11. Prashant Y, et al. Ijppr.Human, 2015; 4: 56-67

12. Mogollón-Morales JA, Nieves E, Rondón M, Rondón-Rivas ME. Propiedad molusquicida de Euphorbia laurifolia A. Juss (Euphorbiaceae) contra Biomphalaria glabrata Say Hospedador Intermediario de Schistosoma mansoni. Avan Biomed 2016; 5: 83-9.

13. González J, Basabe P, Sexmero M, Sánchez Isidoro. Diterpenes From the Latex of Euphorbia broteri.Phytochemistry 1988; 7: 207-212.

14. Smith I. Chromatographic and Electrophoretic Techniques. Zone Electrophoresis. 1960; 2: 200-215

15. Ferreira-Dias S, Valente D, Abreu J. Pattern Recognition of Acorns from Different Quercus Species Based on Oil Content and Fatty Acid Profile. 2003; 54.

16. Wagner H, Blant S. Análisis de Medicamentos de plantas. 2. ${ }^{a}$ ed. Atlas de Cromatografía en capa fina. Springer. 1996: 315-350

17. Finney D, Probit analysis. 3. ${ }^{a}$ ed.,Cambridge University Press, UK. 1971; 1: 76-80.

18. Jaramillo B, Olivero J, Muñoz K. Composición química volátil y toxicidad aguda $(\mathrm{Cl} 50)$ frente a Artemia salina del aceite esencial del Croton malambo colectado en la costa norte colombiana. Scientia et technica. 2007; 1: 299-302 
19. Ospina V, Mantilla J, Conde C, Escobar P. Permeación en piel humana de una nanoemulsión de ftalocianina de aluminio clorada para la optimización de tratamientos tópicos de leishmaniasis cutánea. Revista Ciencias de la Salud, 12(2), 2014: 195-211.

20. Chudzik M, Korzonek-Szlacheta I, Król W. Triterpenos como compuestos potencialmente citotóxicos. Molecules. 2015; 20(1): 1610-1625

21. Zhao H-C, Wang, J-B. The Accumulation of Phytoalexin in Cucumber Plant After Stress. Colloids and Surfaces B: Biointerfaces. 2005: 187-193.

22. Borroto J, Trujillo R, De la Torre Y, Waksman N, Hernández M y Salazar R. Actividad antimicrobiana y toxicidad frente a Artemia salina del extracto diclorometánico de raíces de Morinda royoc L. Revista Cubana de Plantas Medicinales. 2011:34-42.

23. Timoleon M, Pascal W, Ramsay S, Raduis M. Uzor P, Zulfiqar A. Terpenoids From the Stem Bark of Neoboutonia macrocalyx (Euphorbiaceae). Phytochemistry Letters 2015; 12: 328-331 